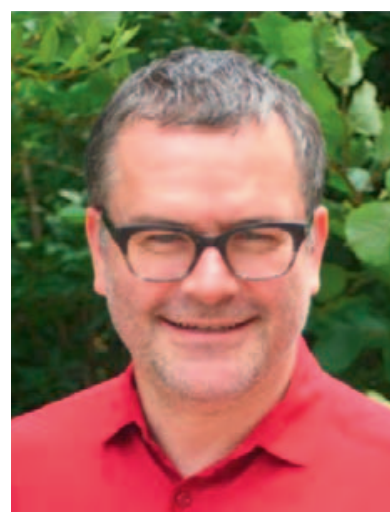

Dr. techn. Herbert Schwabl, Präsident SVKH, Bern, Schweiz

\section{Schlussabstimmung des Heilmittelgesetzes: Eine erste Einschätzung aus Sicht der Komplementär- und Phytoarzneimittel}

Das Parlament hat am 18. März 2016 das revidierte Heilmittelgesetz (HMG) angenommen. Die Referendumsfrist dürfte am 7. Juli ungenutzt verstreichen. Bis zum Ende der Debatte stand die Annahme des Gesetzes wegen der Frage der zulässigen Rabatte auf der Kippe. Erst in der siebten und letzten Runde der Differenzbereinigung zwischen den beiden Räten, in der sogenannten Einigungskonferenz, konnte ein Kompromiss gefunden werden. Die zweite ordentliche Revision des HMG ist für Komplementär- und Phytoarzneimittel ein grosser Erfolg. Neu gilt nicht nur der Grundsatz, dass Komplementär- und Phytoarzneimittel vereinfacht zugelassen werden müssen. Vielmehr führt das Gesetz für alle Kategorien die effektiven Zulassungsvereinfachungen auf. Damit erhält Swissmedic endlich klare Leitplanken, welche der Schweizerische Verband für komplementärmedizinische Heilmittel (SVKH) seit 10 Jahren fordert. Die Grundzüge der Vereinfachungen pro Kategorie werden von SVKH-Geschäftsführer Walter Stüdeli ab Seite 130 der vorliegenden Ausgabe der Schweizerischen ZEITSCHRIFT FÜR GANZHEITSMEDIZIN vorgestellt.

Dr. Herbert Schwabl ist Verwaltungsratspräsident der Padma AG, die in der Schweiz tibetische Arzneimittel herstellt. Mit Padmed Circosan und Padmed Laxan vertreibt die Padma AG zwei Produkte, die von der Spezialitätenliste vergütet werden.
Mit dem Verfassungsartikel 118a Komplementärmedizin geht die Forderung einher, der Schatz an natürlichen Arzneimitteln sei zu wahren und zu fördern. Das neue HMG schafft die Grundlage, dass diese Forderung auch umgesetzt werden kann. Namentlich die Zahl der zugelassenen Phytoarzneimittel war in den letzten Jahren rückgängig. Die Natur bietet einen enormen Arzneimittelschatz, der ungenutzt bleibt. Sinken die Zulassungshürden, so werden Innovationen hoffentlich wieder möglich.

Die Ratsdebatte startete schleppend, der Gesetzesentwurf des Bundesrates war in Bezug auf die Komplementärund Phytoarzneimittel ungenügend. So mussten im ersten Rat rund 10 Änderungsanträge eingereicht werden. Erfreulich ist: Mit einer Ausnahme wurden alle Änderungsanträge angenommen. Dies, obwohl die Widerstände beträchtlich waren. Neben einigen bürgerlichen Kommissionsmitgliedern haben Swissmedic und das Bundesamt für Gesundheit (BAG) während der Beratung in den Gesundheitskommissionen der Komplementärmedizin gegenüber eine kritische Position vertreten. Mit grosser Vehemenz wurde um jede Formulierung gekämpft. Gegen die Zulassungsvereinfachungen wurden Sicherheitsbedenken ins Feld geführt, die dem Gefährdungspotenzial aber in keiner Weise entsprachen. Glücklicherweise hat sich das Parlament nicht auf die Expertenmeinung der Verwaltung verlassen. Erfreulich ist: Die Mehrheit ist den Antragstellern und somit den Positionen des SVKH gefolgt.

Mit dem Abschluss der HMG-Revision endet ein 10-jähriger Lobbying-Prozess im Bundesparlament. Viele Ratsmitglieder, die sich in der parlamentarischen Gruppe Komplementärmedizin versammelt haben, tragen zu diesem Erfolg bei. An erster Stelle gilt der Dank vier Personen: Die ehemalige Nationalrätin Marianne Kleiner (FDP Appenzell Ausserrhoden) reichte 2007 eine parlamentarische Initiative ein, welche die Grundzüge der heutigen Regelung skizzierte. Unermüdlich unterstützt haben uns das Co-Präsidium der parlamentarischen Gruppe, Ständerat Joachim Eder (FDP Zug) und Edith Graf-Litscher (SP Thurgau). Mit grosser Kompetenz und politischem Geschick hat die Komplementärmedizinerin Yvonne Gilli (Grüne St. Gallen) in der Gesundheitskommission entscheidend wichtige Überzeugungsarbeit geleistet und Kompromisse ermöglicht.

Die erfolgreiche Schlussabstimmung im Parlament ist erst ein Etappensieg. Als Nächstes wird das BAG die Verordnungen ausarbeiten. Bei den Komplementär- und Phytoarzneimitteln ist der Ermessensspielraum gering. Das war das erklärte Ziel des SVKH. Seien wir ehrlich: Das Ge-

\section{KARGER}

() 2016 S. Karger GmbH, Freiburg
Dr. techn. Herbert Schwabl

Präsident Schweizerischer Verband für komplementärmedizinische Heilmittel (SVKH) c/o Köhler, Stüdeli \& Partner GmbH

Amthausgasse 18, 3011 Bern, Schweiz

h.schwabl@padma.ch 
setz enthält neu detaillierte Vorgaben, die an sich auf die Stufe einer Verordnung gehören. Der SVKH hat sich für einen hohen Detaillierungsgrad im HMG ausgesprochen, damit der Auftrag des Gesetzgebers klar und deutlich umschrieben wird. Ob die Verordnungen dem Willen des Gesetzgebers entsprechen und ob diese praxistauglich ausgestaltet sind, werden wir im Rahmen der Vernehmlassungen prüfen können, die 2017 gestartet werden. Die Inkraftsetzung des HMG und aller Verordnungen ist im zweiten Semester 2018 geplant.

\section{Vergütung über die Grundversicherung gefährdet}

Zwei Voraussetzungen müssen erfüllt sein, damit die von der Verfassung her geforderte Arzneimittelvielfalt und Therapiefreiheit erhalten bleiben. Erstens müssen Komplementär- und Phytoarzneimittel effektiv vereinfacht zugelassen werden. Diese Forderung dürfte erfüllt sein, sobald die zweite ordentliche Revision des HMG in Kraft tritt. Zweitens muss der Zugang zu Komplementär- und Phytoarzneimitteln gewährt werden und die Preise müssen die Kostenrealität berücksichtigen. Das BAG teilt diese Einschätzung. Es hat dem SVKH am 24. Juli 2015 geschrieben: «Der Verfassungsgeber hat mit Art. 118a Bundesverfassung entschieden, dass sowohl ärztliche komplementärmedizinische Leistungen wie auch die mit der Therapie verbundenen komplementärmedizinischen Arzneimittel von der sozialen Krankenversicherung übernommen werden.»

So weit, so gut. Problematisch ist aber, dass das BAG die Preise für Phytopräparate mehrfach gesenkt hat. Viele Preise der Spezialitätenliste sind heute so niedrig, dass sie nur mit Quersubventionierungen gedeckt werden können. Erschwerend kommt hinzu, dass das BAG bei Homöopathika und Anthroposophika einen Praxiswechsel vollziehen will, ohne dass die Verordnung diesbezüglich angepasst wurde. Homöopathika und Anthroposophika ohne Indi- kationsangabe sollen neu mit pflanzlichen Präparaten mit Indikation verglichen werden. Der SVKH erachtet dies als klaren Verstoss gegen Artikel 34 der Krankenpflege-Leistungsverordnung (KLV). Dieser verlangt, dass die Wirksamkeit «im Verhältnis zu anderen Arzneimitteln gleicher Indikation oder ähnlicher Wirkungsweise» zu prüfen sei. Erstens sind Arzneimittel ohne Indikation aus logischen Gründen nicht mit anderen Arzneimitteln mit Indikation vergleichbar. Zweitens ist die «Wirkungsweise» eines Homöopathikums nicht vergleichbar mit einem wirkstoffbasierten Phytopräparat.

Die Praxisänderung des BAG dürfte gegen einen wichtigen Rechtsgrundsatz verstossen: Gleiches wird mit Gleichem, Ungleiches mit Ungleichem verglichen. Die Folge wäre, dass die Preise der Arzneimittel in der Spezialitätenliste (SL) 70.01 gesenkt statt erhöht würden. Damit könnten diese Leistungen nicht mehr solidarisch über die Grundversicherung vergütet werden.

\section{Nächste Schritte}

Der SVKH hat das BAG mehrfach aufgefordert, in der SL angemessene Preise zu vergüten, welche die Arzneimittelvielfalt und damit die Therapiefreiheit erhalten, den $\mathrm{Zu}-$ gang zu Komplementär- und Phytoarzneimitteln in der SL sicherstellen und den Produktionsstandort für die kleineren und mittleren Unternehmen nicht gefährden.

Bietet das BAG nicht Hand für eine praxistaugliche Verordnungsanwendung, so braucht es zum Schutz der Arzneimittelvielfalt und zur Sicherung der Arbeitsplätze wohl eine Neuregelung. Offen ist, ob diese auf Stufe der Verordnung oder im Krankenversicherungsgesetz erfolgen soll. Der SVKH führt derzeit Gespräche mit dem Gesundheitsdepartement und Mitgliedern der Gesundheitskommission, um eine faire Lösung zu finden. Die Erfahrungen mit dem HMG haben gezeigt, dass das Bundesparlament gewillt ist, den Forderungen des SVKH Gehör zu schenken. 